\title{
Model-Based Predictive Control Applied to Multi-Carrier Energy Systems
}

\author{
Michèle Arnold, Student Member, IEEE, Rudy R. Negenborn, \\ Göran Andersson, Fellow, IEEE, Bart De Schutter, Member, IEEE
}

\begin{abstract}
The optimal operation of an integrated electricity and natural gas infrastructure is investigated. The couplings between the electricity system and the gas system are modeled by so-called energy hubs, which represent the interface between the loads on the one hand and the transmission infrastructures on the other. To increase reliability and efficiency, storage devices are present in the multi-carrier energy system. In order to optimally incorporate these storage devices in the operation of the infrastructure, the capacity constraints and dynamics of these have to be taken into account explicitly. Therefore, we propose a model predictive control approach for controlling the system. This controller takes into account the present constraints and dynamics, and in addition adapts to expected changes of loads and/or energy prices. Simulations in which the proposed scheme is applied to a three-hub benchmark system are presented.
\end{abstract}

Index Terms-Optimal power flow, optimization, model predictive control, electric power systems, natural gas systems

\section{INTRODUCTION}

$\mathbf{N}$ OWADAYS, conventional infrastructures, such as electricity, hydrogen, natural gas, and local district heating systems, are mostly planned and operated independently of each other. In practice, however, these individual systems are coupled, as, e.g., small-scale combined heat and power plants $(\mu \mathrm{CHP})$ and other distributed generation plants (so-called coand trigeneration [1]) are used more and more. It is therefore expected that by pursuing the integrated control of several of such systems, improved efficiency and performance can be achieved. The various energy carriers available and the conversion possible between them significantly affect both the technical and the economical operation of energy systems. In particular, consumers get flexibility in supply and could therefore decide in favor of, e.g., cost, reliability, system emissions, availability, or a combination of these.

Currently, research effort is addressing the integrated control of combined electricity and natural gas systems [2], [3]. In [2], the impact of natural gas infrastructures contingencies on the operation of electric power systems has been analyzed. In [3], the electricity and gas systems are coupled by using the concept of so-called energy hubs [4]. The energy hubs serve

M. Arnold (corresponding author) and G. Andersson are with the Power Systems Laboratory, ETH Zürich, Physikstrasse 3, 8092 Zürich, Switzerland, e-mail: $\{$ arnold, andersson $\}$ @eeh. ee. ethz . ch. R.R. Negenborn and B. De Schutter are with the Delft Center for Systems and Control of the Delft University of Technology, Mekelweg 2, 2628 CD Delft, The Netherlands, e-mail: r.r.negenbornatudelft.nl, b. deschutteradcsc.tudelft.nl. B. De Schutter is also with the Marine and Transport Technology department of the Delft University of Technology.

978-1-4244-4241-6/09/\$25.00 (C2009 IEEE as interface between the loads and the transmission infrastructures of both types of systems. The above mentioned twocarrier system is then modeled as a number of interconnected energy hubs.

Because of the increasing number of facilities for distributed generation with fluctuating energy infeed (generation profiles), the issue of storing energy becomes more important. Electric energy storage devices are expensive and their operation causes energy losses. In order to still enable the electric energy supply in time, the operation of a $\mu \mathrm{CHP}$ device in combination with a heat storage is studied. Since the ratio of produced heat and electricity of a $\mu \mathrm{CHP}$ device is predefined, its operation always has to be aligned with the electric and thermal loads. By means of the heat storage, the $\mu \mathrm{CHP}$ device can be operated with focus on following the electric load while storing the thereby produced heat. The operation costs are presented when comparing the operation of the $\mu \mathrm{CHP}$ device with and without heat storage support. Furthermore, the costs are compared with the decoupled operation mode, i.e. when the electricity and natural gas system are operated independently of each other, i.e. when no $\mu$ CHP devices are in usage.

For determining the optimal operation of the integrated electricity and natural gas system an optimal power flow problem is solved. An optimization problem for the entire system is solved by a central controller, which determines the optimal operational set-points of the system, i.e. of the energy generation units, converters and storage devices. In [3], a steady-state optimization has been solved, in which the interconnected energy hub system is modeled with static, steady-state models. In this paper, storage devices with dynamic behavior are present in the multi-carrier system. Since they cause a dependency between consecutive time steps optimization over multiple time steps is required. Therefore, for the optimal operation of the system, actions have to be determined taking the expected future behavior of the system into account. For optimizing the operation over multiple time steps, we propose to use model predictive control (MPC) [5], [6]. MPC is widely used in different application areas since system dynamics, data forecasts and operational constraints can be taken into account explicitly. In our case, a supervisory coordinator uses MPC to determine the actions for the individual energy hubs that give the best predicted behavior, based on characteristics of the transmission infrastructures, the dynamics of the storage devices, and the load and price profiles. By using this predictive approach, the energy usage can be adapted to expected fluctuations in the energy prices, 
as well as to expected changes in the load profiles.

This paper is organized as follows. In Section II the mathematical model of the considered two-carrier system is given. MPC is proposed in Section III for the optimal operation of the system. The control problem for the network is presented, taking future system behavior into account. Simulations in which the proposed method is applied to a three-hub benchmark system are presented in Section IV. Section V concludes this paper and outlines directions for future research.

\section{MODELING}

The studied multi-carrier energy systems consist of several interconnected energy hubs. As example we consider a system consisting of three hubs that are interconnected by an electricity and natural gas transmission system (Fig. 1). The couplings between the electricity and the gas system are modeled by means of energy hubs. In principle, an energy hub is a generalization or extension of a network node that includes conversion, conditioning, and storage of multiple energy carriers. It represents the interface between the energy sources and transmission lines on the one hand and the power consumers on the other hand. The energy hub is a modeling concept with no restrictions to the size of the modeled system. Single power plants or industrial buildings as well as bounded geographical areas such as entire towns can be modeled as energy hubs. Below we formalize the model of this system.

\section{A. System setup}

In the system under study, each energy hub represents a general consumer, e.g., a household, that uses both electricity and gas. Each of the hubs has its own local electrical energy production $\left(\mathrm{G}_{i}\right.$, with electric power production $P_{\mathrm{e}, i}^{\mathrm{G}}$, for $i \in$ $\{1,2,3\})$. Hub $\mathrm{H}_{1}$ is connected to a large gas network $\mathrm{N}_{1}$, with gas infeed $P_{\mathrm{g}, 1}^{\mathrm{G}}$. In addition, hub $\mathrm{H}_{2}$ can obtain gas from a smaller local gas tank, modeled as gas infeed $P_{\mathrm{g}, 2}^{\mathrm{G}}$. Each hub consumes electric power $P_{\mathrm{e}, i}^{\mathrm{H}}$ and gas $P_{\mathrm{g}, i}^{\mathrm{H}}$, and supplies energy to its electric load $L_{\mathrm{e}, i}$ and its heat load $L_{\mathrm{h}, i}$. The hubs contain converter and storage devices in order to fulfill their energy load requirements. For energy conversion, the hubs contain a $\mu \mathrm{CHP}$ device and a furnace. The $\mu \mathrm{CHP}$ device couples the two energy systems as it simultaneously produces electricity and heat from natural gas. All hubs additionally comprise a hot water storage device. Compressors $\left(\mathrm{C}_{i j}\right.$, for $(i, j) \in$ $\{(1,2),(1,3)\})$ are present in the gas network within the pipelines originating from hub $\mathrm{H}_{1}$. The compressors provide a pressure decay and enable the gas flow from the large gas network to the surrounding gas sinks.

Depending on the prices and load profiles, the $\mu \mathrm{CHP}$ device is utilized differently. At high electricity prices, the $\mu \mathrm{CHP}$ device is mainly operated according to the electric load. The thereby produced heat is then either used to supply the thermal load or stored in the heat storage device. At low electricity prices, the electric load is preferably supplied directly by the electricity network and the gas is used for supplying the thermal load via the furnace. Hence, there are several ways in which electric and thermal load demands can be fulfilled. This redundancy increases the reliability of supply and at the same

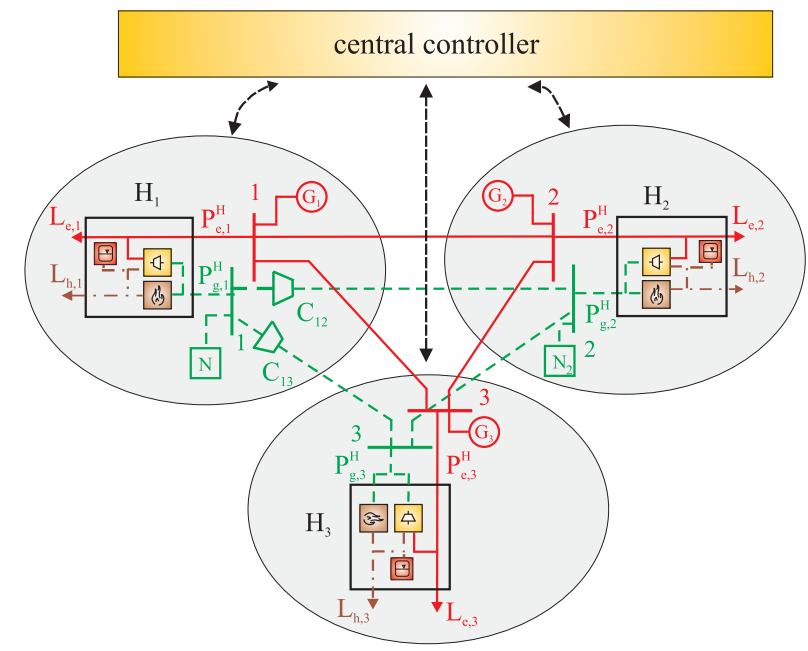

Fig. 1. System setup of three interconnected energy hubs. Active power is provided by generators $\mathrm{G}_{1}, \mathrm{G}_{2}, \mathrm{G}_{3}$. Hubs $\mathrm{H}_{1}$ and $\mathrm{H}_{2}$ have access to adjacent natural gas networks $\mathrm{N}_{1}, \mathrm{~N}_{2}$. Central controller measures all system variables (dashed arrows).

time provides the possibility for optimizing the input energies, e.g., using criteria such as cost, availability, emissions, etc. [4].

In the following equations, time is discretized into discrete time steps $k=0,1, \ldots$, where a discrete time step $k$ corresponds to the continuous time $k T$, with $T$ being the sampling time in hours.

\section{B. Power conversion}

For each of the three energy hubs, the electric load $L_{\mathrm{e}, i}(k)$ and the heat load $L_{\mathrm{h}, i}(k)$ at a time step $k$ are related to the electricity $P_{\mathrm{e}, i}^{\mathrm{H}}(k)$ and gas hub input $P_{\mathrm{g}, i}^{\mathrm{H}}(k)$ as follows:

$$
\begin{aligned}
& \underbrace{\left[\begin{array}{c}
L_{\mathrm{e}, i}(k) \\
L_{\mathrm{h}, i}(k)
\end{array}\right]}_{\mathbf{L}_{\mathbf{i}}(k)}= \\
& \underbrace{\left[\begin{array}{cc}
1 & \nu_{\mathrm{g}, i}(k) \eta_{\mathrm{g}, \mathrm{e}, i}^{\mathrm{CHP}} \\
0 & \nu_{\mathrm{g}, i}(k) \eta_{\mathrm{g}, \mathrm{h}, i}^{\mathrm{CHP}}+\left(1-\nu_{\mathrm{g}, i}(k)\right) \eta_{\mathrm{g}, \mathrm{h}, i}^{\mathrm{F}}
\end{array}\right]}_{\mathbf{C}_{\mathbf{i}}(k)} \underbrace{\left[\begin{array}{c}
P_{\mathrm{e}, i}^{\mathrm{H}}(k) \\
P_{\mathrm{g}, i}^{\mathrm{H}}(k)
\end{array}\right]}_{\mathbf{P}_{\mathbf{i}}(k)},
\end{aligned}
$$

where $\eta_{\mathrm{g}, \mathrm{e}, i}^{\mathrm{CHP}}$ and $\eta_{\mathrm{g}, \mathrm{h}, i}^{\mathrm{CHP}}$ denote the gas-electric and gas-heat efficiencies of the $\mu \mathrm{CHP}$ device ${ }^{1}$ and where $\eta_{\mathrm{g}, \mathrm{h}, i}^{\mathrm{F}}$ denotes the efficiency of the furnace. The variable $\nu_{\mathrm{g}, i}(k)\left(0 \leq \nu_{\mathrm{g}, i}(k) \leq\right.$ 1) represents a dispatch factor that determines how the gas is divided between the $\mu \mathrm{CHP}$ and the furnace. The term $\nu_{\mathrm{g}, i}(k) P_{\mathrm{g}, i}^{\mathrm{H}}(k)$ defines the gas input power fed into the $\mu \mathrm{CHP}$ and the part $\left(1-\nu_{\mathrm{g}, i}(k)\right) P_{\mathrm{g}, i}^{\mathrm{H}}(k)$ defines the gas input power going into the furnace. In general, the conversion between the input and output powers, collected in vectors $\mathbf{P}_{\mathrm{i}}(k)$ and $\mathbf{L}_{\mathrm{i}}(k)$, respectively, is expressed by a coupling matrix $\mathbf{C}_{\mathrm{i}}(k)$. As the dispatch factor $\nu_{\mathrm{g}, i}(k)$ is variable, different input vectors $\mathbf{P}_{\mathrm{i}}(k)$ can be found to fulfill the output loads $\mathbf{L}_{\mathrm{i}}(k)$. This offers additional degrees of freedom in supply.

\footnotetext{
${ }^{1}$ In this paper the efficiencies are assumed to be constant. However, they can also be dependent on, e.g., the converted power level.
} 


\section{Power storage}

The storage device is modeled as an ideal storage in combination with a storage interface. In the considered setup, hot water storage devices are implemented. The relation between the heat power exchange $M_{\mathrm{h}, i}(k)$ and the effectively stored energy $E_{\mathrm{h}, i}(k)$ at time step $k$ is defined by the following equation:

$$
\begin{aligned}
M_{\mathrm{h}, i}(k) & =\frac{\dot{E}_{h}}{e_{\mathrm{h}, i}}=\frac{1}{e_{\mathrm{h}, i}} \frac{d E_{h, i}}{d t} \approx \frac{1}{e_{\mathrm{h}, i}} \frac{\Delta E_{h, i}}{\Delta t} \\
& =\frac{1}{e_{\mathrm{h}, i}}\left(E_{\mathrm{h}, i}(k)-E_{\mathrm{h}, i}(k-1)+E_{\mathrm{h}, i}^{\mathrm{stb}}\right),
\end{aligned}
$$

with

$$
e_{\mathrm{h}, i}=\left\{\begin{array}{lll}
e_{\mathrm{h}, i}^{+} & \text {if } M_{\mathrm{h}, i}(k) \geq 0 & \text { (charging/ standby) } \\
1 / e_{\mathrm{h}, i}^{-} & \text {else } & \text { (discharging) }
\end{array}\right.
$$

where $e_{\mathrm{h}, i}^{+}, e_{\mathrm{h}, i}^{-}$are the charging and discharging efficiency of the heat storage device, including the efficiency of the storage interface, converting the energy carrier exchanged with the system into the carrier stored internally. $E_{\mathrm{h}, i}(k)$ denotes the storage energy at time step $k$. $E_{\mathrm{h}, i}^{\mathrm{stb}}$ represents the standby energy losses of the heat storage device per period $\left(E_{\mathrm{h}, i}^{\mathrm{stb}} \geq 0\right)$. For each hub hot water storage devices are implemented. Equation (1) is therefore completed with additional storage power flows, which are collected in a vector $\mathbf{M}_{\mathbf{i}}(k)$ :

$$
\begin{aligned}
& \underbrace{\left[\begin{array}{c}
L_{\mathrm{e}, i}(k) \\
L_{\mathrm{h}, i}(k)+M_{\mathrm{h}, i}(k)
\end{array}\right]}_{\mathbf{L}_{\mathbf{i}}(k)+\mathbf{M}_{\mathbf{i}}(k)}= \\
& \underbrace{\left[\begin{array}{cc}
1 & \nu_{\mathrm{g}, i}(k) \eta_{\mathrm{g}, \mathrm{e}, i}^{\mathrm{CHP}} \\
0 & \nu_{\mathrm{g}, i}(k) \eta_{\mathrm{g}, \mathrm{h}, i}^{\mathrm{CHP}}+\left(1-\nu_{\mathrm{g}, i}(k)\right) \eta_{\mathrm{g}, \mathrm{h}, i}^{\mathrm{F}}
\end{array}\right]}_{\mathbf{C}_{\mathbf{i}}(k)} \underbrace{\left[\begin{array}{c}
P_{\mathrm{e}, i}^{\mathrm{H}}(k) \\
P_{\mathrm{g}, i}^{\mathrm{H}}(k)
\end{array}\right]}_{\mathbf{P}_{\mathbf{i}}(k)} .
\end{aligned}
$$

The storage power flows $\mathbf{M}_{\mathrm{i}}(k)$ define how the storage energies affect the output flows, i.e., describe how the storage energy derivatives are mapped into equivalent output-side flows.

\section{Transmission network}

For the transmission networks of both the electricity network and the gas pipeline network, power flow models based on nodal power balances are implemented. Analogous to the hub equations, the nodal power balances are defined per time step $k$ as well.

a) AC electricity network: Electric power flows are formulated as nodal power balances of the complex power, according to the normal power flow equations [7]. At node $m$, the complex power balance at time step $k$ is stated as

$$
S_{m}(k)-\sum_{n \in N_{m}} S_{m n}(k)=0
$$

where $S_{m}(k)$ is the complex power injected at node $m$. $S_{m n}(k)$ denotes the power flow to all adjacent nodes $n$ of node $m$, summarized in the set $N_{m}$. The line flows are expressed

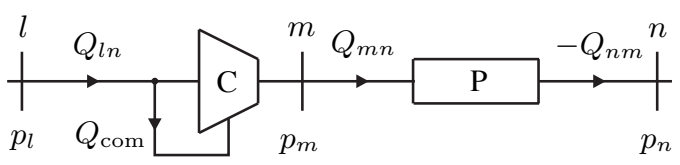

Fig. 2. Model of a gas pipeline with compressor (C) and pipeline (P). Compressor demand is modeled as additional power flow $Q_{\mathrm{com}}$.

by the voltage magnitudes $V(k)$ and angles $\theta(k)$ and the line parameters:

$$
\begin{array}{r}
S_{m n}(k)=y_{m n}^{*} V_{m}(k) e^{j \theta_{m}(k)}\left(V_{m}(k) e^{-j \theta_{m}(k)}\right. \\
\left.-V_{n}(k) e^{-j \theta_{n}(k)}\right)-j b_{m n}^{\text {sh }} V_{m}(k)^{2}
\end{array}
$$

where the superscript ${ }^{*}$ denotes the conjugate complex of the value. The line is modeled as $\pi$-equivalent with the shunt admittance $y_{m n}$ and the shunt susceptance $b_{m n}^{\mathrm{sh}}$.

b) Pipeline network: Figure 2 shows the model of a gas pipeline composed of a compressor and a pipeline element. The volume flow balance at node $m$ at time step $k$ is defined as

$$
Q_{m}(k)-\sum_{n \in \mathcal{N}_{m}} Q_{m n}(k)=0,
$$

where $Q_{m}(k)$ is the volume flow injected at node $m, Q_{m n}(k)$ denotes the line flow between nodes $m$ and $n$, and $\mathcal{N}_{m}$ denotes the set of neighboring nodes of node $m$, i.e., the nodes connected to node $m$ through a pipeline. The line flow $Q_{m n}(k)$ is defined as

$$
Q_{m n}(k)=k_{m n} s_{m n} \sqrt{s_{m n}\left(p_{m}(k)^{2}-p_{n}(k)^{2}\right)},
$$

where $p_{m}(k)$ and $p_{n}(k)$ denote the upstream and downstream pressures, respectively, and $k_{m n}$ identifies the line constant. The variable $s_{m n}$ indicates the direction of the gas flow as

$$
s_{m n}= \begin{cases}+1 & \text { if } p_{m}(k) \geq p_{n}(k) \\ -1 & \text { otherwise }\end{cases}
$$

To maintain a certain pressure level a compressor is needed. Here, the compressor is driven by a gas turbine which is modeled as additional gas flow

$$
Q_{\text {com }}(k)=k_{\text {com }} Q_{m n}(k)\left(p_{m}(k)-p_{l}(k)\right),
$$

where $p_{l}(k)$ denotes the pressure at the compressor input side and $k_{\text {com }}$ is a compressor constant. The resulting gas flow into the pipeline $Q_{m n}(k)$ is therefore determined by

$$
Q_{m n}(k)=Q_{l n}(k)-Q_{\text {com }}(k) .
$$

The pressure at the compressor output $p_{m}(k)$ is determined by

$$
p_{m}(k)=p_{\text {inc }}(k) p_{l}(k),
$$

where $p_{\text {inc }}(k)$ defines the pressure amplification of the compressor. Depending on the required line flow $Q_{m n}(k), p_{\text {inc }}(k)$ is adjusted accordingly. For the purpose of this study, these simplified compressor models provide sufficient accuracy. More advanced compressor equations taking into account changing fluid properties are given in [8]. 


\section{E. Combined energy hub transmission network modeling}

The combined electricity and gas network is obtained by combining the power flow models stated above. For each time step $k$ an algebraic state vector $\mathbf{z}(k)$ and a dynamic state vector $\mathbf{x}(k)$ are defined. The algebraic state vector includes the variables for which no dynamics are explicitly defined. The dynamic state vector includes variables for which dynamics are included. Hence,

$$
\begin{aligned}
\mathbf{x}(k) & =\left[\mathbf{E}_{\mathrm{h}}(k)\right]^{\mathrm{T}} \\
\mathbf{z}(k) & =\left[\mathbf{V}^{\mathrm{T}}(k) \boldsymbol{\theta}^{\mathrm{T}}(k) \mathbf{p}^{\mathrm{T}}(k) \mathbf{p}_{\text {inc }}^{\mathrm{T}}(k)\right. \\
&
\end{aligned}
$$

where

- $\mathbf{V}(k)=\left[V_{1}(k), V_{2}(k), V_{3}(k)\right]^{\mathrm{T}}$ and $\boldsymbol{\theta}(k)=$ $\left[\theta_{1}(k), \theta_{2}(k), \theta_{3}(k)\right]^{\mathrm{T}}$ denote the voltage magnitudes and angles of the electric buses, respectively,

- $\mathbf{p}(k)=\left[p_{1}(k), p_{2}(k), p_{3}(k)\right]^{\mathrm{T}}$ denotes the nodal pressures of all gas buses,

- $\mathbf{p}_{\text {inc }}(k)=\left[p_{\text {inc }, 1}(k), p_{\text {inc }, 2}(k)\right]^{\mathrm{T}}$ denotes the pressure amplification of the compressors,

- $\mathbf{P}_{\mathrm{e}}^{\mathrm{H}}(k)=\left[P_{\mathrm{e}, 1}^{\mathrm{H}}(k), P_{\mathrm{e}, 2}^{\mathrm{H}}(k), P_{\mathrm{e}, 3}^{\mathrm{H}}(k)\right]^{\mathrm{T}}$ denotes the electric inputs of the hubs, and

- $\mathbf{P}_{\mathrm{g}}^{\mathrm{H}}(k)=\left[P_{\mathrm{g}, 1}^{\mathrm{H}}(k), P_{\mathrm{g}, 2}^{\mathrm{H}}(k), P_{\mathrm{g}, 3}^{\mathrm{H}}(k)\right]^{\mathrm{T}}$ denotes the gas inputs of the hubs and

- $\mathbf{E}_{\mathrm{h}}(k)=\left[\mathbf{E}_{\mathrm{h}, 1}(k), \mathbf{E}_{\mathrm{h}, 2}(k), \mathbf{E}_{\mathrm{h}, 3}(k)\right]^{\mathrm{T}}$ denotes the energy contents of the heat storage devices.

At each time step $k$, the control variables $\mathbf{u}(k)$ are defined to include the active power generation of all generators, the natural gas imports of all gas networks and the dispatch factors of all hubs, i.e.,

$$
\mathbf{u}(k)=\left[\begin{array}{lll}
\left(\mathbf{P}_{\mathrm{e}}^{\mathrm{G}}\right)^{\mathrm{T}}(k) & \left(\mathbf{P}_{\mathrm{g}}^{\mathrm{G}}\right)^{\mathrm{T}}(k) & \boldsymbol{\nu}_{\mathrm{g}}^{\mathrm{T}}(k)
\end{array}\right]^{\mathrm{T}},
$$

where

- $\mathbf{P}_{\mathrm{e}}^{\mathrm{G}}(k)=\left[P_{\mathrm{e}, 1}^{\mathrm{G}}(k), P_{\mathrm{e}, 2}^{\mathrm{G}}(k), P_{\mathrm{e}, 3}^{\mathrm{G}}(k)\right]^{\mathrm{T}}$ denotes the active power generation of all generators

- $\mathbf{P}_{\mathrm{g}}^{\mathrm{G}}(k)=\left[P_{\mathrm{g}, 1}^{\mathrm{G}}(k), P_{\mathrm{g}, 2}^{\mathrm{G}}(k)\right]^{\mathrm{T}}$ defines the natural gas imports and

- $\boldsymbol{\nu}_{\mathrm{g}}(k)=\left[\nu_{\mathrm{g}, 1}(k), \nu_{\mathrm{g}, 2}(k), \nu_{\mathrm{g}, 3}(k)\right]^{\mathrm{T}}$ describes the dispatch factors at the gas input junctions.

Now, the model that we use to represent the combined electricity and gas network, including the hub equations with the dynamics, can be written in compact form as

$$
\begin{aligned}
\mathbf{x}(k+1) & =\mathbf{f}(\mathbf{x}(k), \mathbf{z}(k), \mathbf{u}(k)) \\
\mathbf{0} & =\mathbf{g}(\mathbf{x}(k), \mathbf{z}(k), \mathbf{u}(k)) .
\end{aligned}
$$

\section{CONTROL PROBLEM}

In this section we discuss the control of the system introduced above. The goal is to implement a supervisory, central controller that defines the set-points of the energy generation units of the hubs. This central controller measures all variables in the network and determines all actions for all actuators. In Fig. 1 the central controller is indicated. The dashed lines indicate the information exchange between the individual hubs and the central controller. It is assumed that within the hubs,

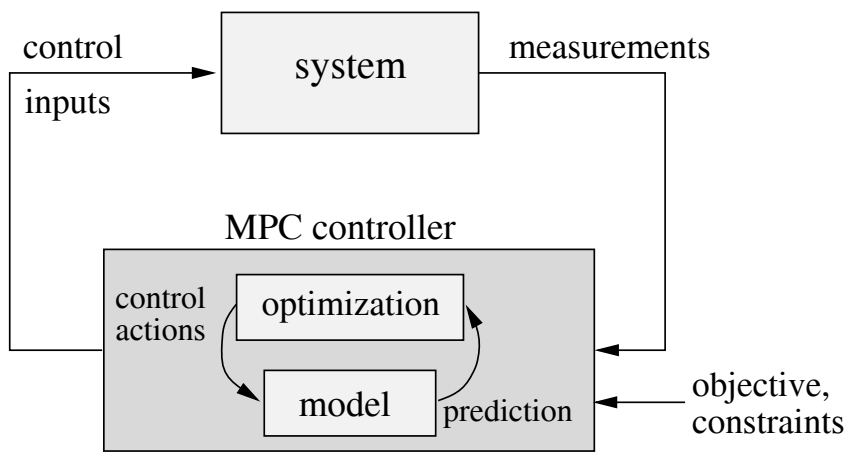

Fig. 3. Illustration of model predictive control, showing interactions between system and controller.

there are local controllers which ensure that the obtained setpoints are met. We propose that the central controller uses an MPC approach.

The goal of the control scheme is to determine values for the control variables $\mathbf{u}(k)$ in such a way that the costs for electricity generation and natural gas usage are minimized over the considered simulation period. Hence, the control problem can be stated as determining the inputs $\mathbf{u}(k)$ in such a way that the control objectives are achieved, while satisfying the system constraints ${ }^{2}$. First, we explain the basic idea of MPC. Then, the control problem for the considered hub system is formulated.

\section{A. Model predictive control}

Since storage devices are included in the optimization, optimization for multiple periods is required. As control strategy we propose to use MPC. MPC [5], [6] is an optimizationbased control strategy. An optimization problem is solved, in which an internal model is used to find those actions that give the best predicted behavior of the system over a predefined prediction horizon with length $N$. In this optimization operational constraints are also taken into account. MPC is suited for control of multi-carrier systems, since it can adequately take into account the dynamics of energy storage devices and the characteristics of the electricity and gas networks. MPC operates in a receding horizon fashion, meaning that at each time step new measurements of the system and new predictions into the future are made and new control actions are computed. By using MPC, actions can be determined that anticipate future events, such as increasing or decreasing energy prices.

In Fig. 3 MPC is illustrated schematically. At each control step $k$, an MPC controller first measures the current state of the system, $\mathbf{x}(k)$. Then, it determines using (numerical) optimization which control input $\mathbf{u}(k)$ to provide by determining the actions that over a prediction horizon of $N$ time steps give the best predicted performance according to a given objective function. The control variables computed for the first prediction step are then applied to the system. The system then transitions to a new state, $\mathbf{x}(k+1)$, after which the cycle is repeated.

\footnotetext{
${ }^{2}$ In addition to the stated objectives, it is straightforward to also include voltage regulation and power flow limitations as control objectives.
} 


\section{B. Control problem formulation}

In the MPC formulation the central controller determines the inputs $\mathbf{u}(k)$ for the network. The control objective is to minimize the energy costs, represented by the following objective function, where costs of the individual energy carriers are modeled as quadratic functions of the corresponding powers:

$$
\begin{aligned}
J=\sum_{l=0}^{N-1} \sum_{i \in G}^{3}\left(q_{i}^{\mathrm{G}}(k+l)\left(P_{\mathrm{e}, i}^{\mathrm{G}}(k+l)\right)^{2}\right) \\
+q_{i}^{\mathrm{N}}(k+l)\left(P_{\mathrm{g}, i}^{\mathrm{G}}(k+l)\right)^{2},
\end{aligned}
$$

where $G$ includes all generation units, i.e., the three generators and the two natural gas imports. The prices for electricity generation of all generators $i$ and for the natural gas consumption of the two gas networks for time step $k$ are denoted by $q_{i}^{\mathrm{G}}(k)$ and $q_{i}^{\mathrm{N}}(k)$, respectively. These prices can vary throughout the day. The central control problem formulation is now stated as

$$
\min _{\tilde{\mathbf{u}}(k)} J(\tilde{\mathbf{x}}(k+1), \tilde{\mathbf{z}}(k), \tilde{\mathbf{u}}(k))
$$

subject to

$$
\begin{aligned}
& \tilde{\mathbf{x}}(k+1)=\tilde{\mathbf{f}}(\tilde{\mathbf{x}}(k), \tilde{\mathbf{z}}(k), \tilde{\mathbf{u}}(k)) \\
& \tilde{\mathbf{g}}(\tilde{\mathbf{x}}(k), \tilde{\mathbf{z}}(k), \tilde{\mathbf{u}}(k))=\mathbf{0} \\
& \tilde{\mathbf{h}}(\tilde{\mathbf{x}}(k), \tilde{\mathbf{z}}(k), \tilde{\mathbf{u}}(k)) \leq \mathbf{0},
\end{aligned}
$$

where the tilde over a variable represents a vector with the values of this variable over a prediction horizon of $N$ steps, e.g., $\tilde{\mathbf{u}}(k)=\left[\mathbf{u}^{\mathrm{T}(k)}, \ldots, \mathbf{u}^{\mathrm{T}(k+N-1)}\right]^{\mathrm{T}}$. The inequality constraints (22) comprise limits on the voltage magnitudes, active and reactive power flows, pressures, changes in compressor settings and dispatch factors. Furthermore, power limitations on the hub inputs and on gas and electricity generation are also incorporated into (22). Regarding the storage devices, limits on the storage contents and the storage flows are imposed.

The optimization problem (19)-(22) is a nonlinear programming problem [9], which can be solved using solvers for nonlinear programming, such as sequential quadratic programming [9]. In general, the solution space is non-convex and therefore finding a global optimum cannot be guaranteed. Only a local optimum is returned by numerical optimization.

\section{CASE STUdY}

Simulations are presented, in which the proposed MPC scheme is applied to the three-hub benchmark system depicted in Fig. 1. We use the snopt solver through the Tomlab interface [10] in Matlab.

\section{A. Simulation setup}

At each hub, the daily profiles of the load demands and the energy prices are known in advance. Here, we assume that these load and price forecasts are perfect. However, in reality, there are always forecast errors. As a first study, we assume perfect forecasts and it is believed that the following results are representative also for small forecast errors. The given profiles are typical profiles for a household. The electricity and heat loads are assumed to be the same for all hubs and are depicted in the upper plot in Fig 4. Regarding the prices, the
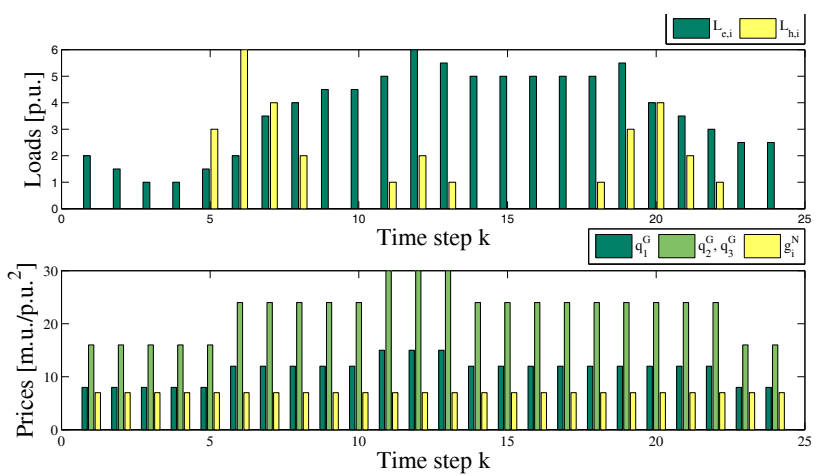

Fig. 4. Daily profile for electricity $L_{\mathrm{e}, i}(k)$ and heat loads $L_{\mathrm{h}, i}(k)$ (upper plot) and prices for electricity $q_{i}^{\mathrm{G}}(k)$ and natural gas consumption $q_{i}^{\mathrm{N}}(k)$ (lower plot).

electricity prices are varying during the day, showing a peak around noon. Furthermore, electricity generation at hubs $\mathrm{H}_{2}$ and $\mathrm{H}_{3}$ is chosen twice as expensive as at hub $\mathrm{H}_{1}$. The reason for choosing different electricity prices is to obtain three hubs with different setups. (Hub $\mathrm{H}_{1}$ has a cheap access to electricity and gas, hub $\mathrm{H}_{2}$ has an expensive electricity and a limited gas access and hub $\mathrm{H}_{3}$ has an expensive electricity access and no gas access.) Gas prices remain constant throughout the day and are the same for all hubs. The loads are given in p.u. (per unit) values and the price coefficients are described in m.u./p.u. ${ }^{2}$, where m.u. refers to monetary units.

Regarding the electricity network, bus 1 is modeled as slack bus, i.e., having the voltage angle and voltage magnitude fixed $\left(V_{1}(k)=1 \angle 0^{\circ}\right.$ p.u.). The other two buses are modeled as PV buses, for which the net active power and the voltage magnitude are specified. Also within the gas network bus 1 serves as slack bus, having a fixed pressure value of 1 p.u. The coefficients and simulation parameters used are listed in Table I. Since hub $\mathrm{H}_{2}$ is assumed to have only access to a smaller gas tank, a flow rate constraint of 2 p.u. is imposed on $P_{\mathrm{g}, 2}^{\mathrm{G}}(k)$. The network is mainly supplied via the large gas network $P_{\mathrm{g}, 1}^{\mathrm{G}}(k)$, delivering the gas to the neighboring buses by means of the two compressors.

\section{TABLE I}

PARAMETERS OF THE THREE-HUB SYSTEM IN P.U.

\begin{tabular}{|l|l|}
\hline \multicolumn{2}{|l|}{ coefficients } \\
\hline \hline$\mu \mathrm{CHP}$ & $\eta_{\mathrm{g}, \mathrm{e}, i}^{\mathrm{CHP}}=0.3, \eta_{\mathrm{g}, \mathrm{h}, i}^{\mathrm{CHP}}=0.4$ \\
\hline $\mathrm{F}$ & $\eta_{\mathrm{g}, \mathrm{h}, i}^{\mathrm{F}}=0.75$ \\
\hline$E_{\mathrm{h}, i}^{\mathrm{stb}}$ & $E_{\mathrm{h}, i}^{\mathrm{sb}}=0.2$ \\
\hline$e_{\mathrm{h}, \mathrm{i}}$ & $e_{\mathrm{h}, \mathrm{i}}^{+}=e_{\mathrm{h}, \mathrm{i}}^{-}=0.9$ \\
\hline \hline limits \\
\hline \hline$V_{\mathrm{i}}$ & $0.9 \leq\left|V_{\mathrm{i}}\right| \leq 1.1$ \\
\hline$P_{\mathrm{e}, i}^{\mathrm{G}}$ & $0 \leq P_{\mathrm{e}, i}^{\mathrm{G}} \leq 10$ \\
\hline$p_{\mathrm{i}}$ & $0.8 \leq p_{\mathrm{i}} \leq 1.2$ \\
\hline$p_{\text {inc }, \mathrm{i}}$ & $1.2 \leq p_{\mathrm{inc}, \mathrm{i}} \leq 1.8$ \\
\hline$\nu_{i}$ & $0 \leq \nu_{i} \leq 1$ \\
\hline$P_{\mathrm{g}, i}^{\mathrm{G}}$ & $0 \leq P_{\mathrm{g}, 1}^{\mathrm{G}} \leq 20,0 \leq P_{\mathrm{g}, 2}^{\mathrm{G}} \leq 2$ \\
\hline$E_{\mathrm{i}}$ & $0.5 \leq E_{\mathrm{i}} \leq 3$ \\
\hline$M_{\mathrm{h}, i}$ & $-3 \leq M_{\mathrm{h}, i} \leq 3$ \\
\hline
\end{tabular}




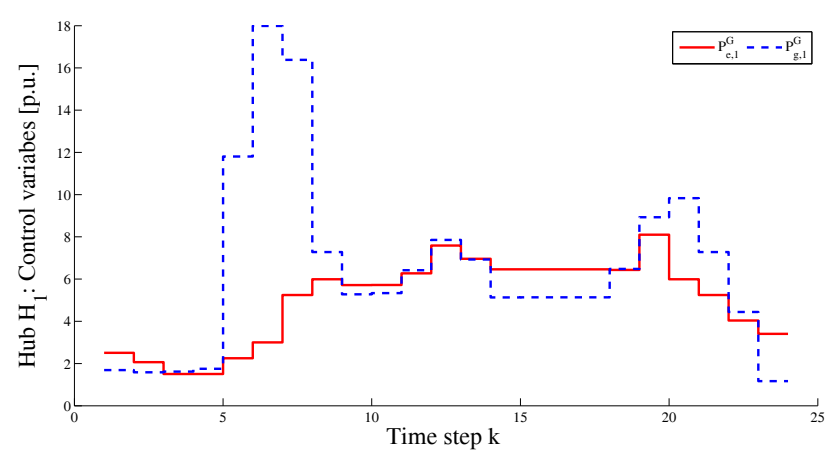

Fig. 5. Active power generation $P_{\mathrm{e}, 1}^{\mathrm{G}}(k)$ and natural gas import $P_{\mathrm{g}, 1}^{\mathrm{G}}(k)$ of hub $\mathrm{H}_{1}$ over entire simulation horizon, for a prediction horizon with length $N=5$.

Based on the profiles, the total generation costs are minimized for a simulation of $N_{\text {sim }}=24$ time steps, where one time step corresponds to 1 hour. To analyze the performance of the central controller, we vary the length of the prediction horizon $N$ between $N=1$, i.e. no prediction (optimizing just for the actual time step), and $N=24$, i.e. predicting for all 24 time steps at once.

\section{B. Prediction horizon with length $N=5$}

The behavior of the system is illustrated for a prediction horizon with length $N=5$. We consider this length of prediction horizon as adequate for practical applications and it represents a proper trade-off between control performance on the one side and obtainable forecasts and computational effort on the other side. An optimization for 5 time steps is run, at each time step $k$ implementing only the control variables for the current time step $k$ and then starting the procedure again at time step $k+1$ using updated system measurements. Running this procedure over the entire simulation period $[0,24]$, total operation costs of $2.73 \cdot 10^{4}$ m.u are obtained. Figure 5 shows the respective evolution of the active power generation and natural gas import at hub $\mathrm{H}_{1}$. The electricity generation mainly corresponds to the electricity load pattern and the natural gas import evolves similar to the heat loads. However, natural gas is also used during time periods, in which no heat is required. During these periods gas is converted by the $\mu \mathrm{CHP}$ device for supporting the electricity generation. The thereby produced heat is stored and used later for the heat supply. At hubs $\mathrm{H}_{2}$ and $\mathrm{H}_{3}$, the active power generation shows a similar evolution, but with a level roughly half as high as at hub $\mathrm{H}_{1}$, since their generation is twice as expensive as at hub $\mathrm{H}_{1}$. At hub $\mathrm{H}_{2}$, where the gas access is limited, the maximum amount of gas is imported during day times, i.e. when the prices for electricity generation are high.

In Fig. 6, the storage contents for different lengths of prediction horizons are presented. The horizontal lines indicate the storage limits ( 0.5 p.u. $\leq E_{i}(k) \leq 3$ p.u.). First, we focus on the storage behavior for a prediction horizon of length $N=5$, which is represented by the dashed line. All three storage devices are half full before the optimization starts. In general, the storage devices are mainly discharged during
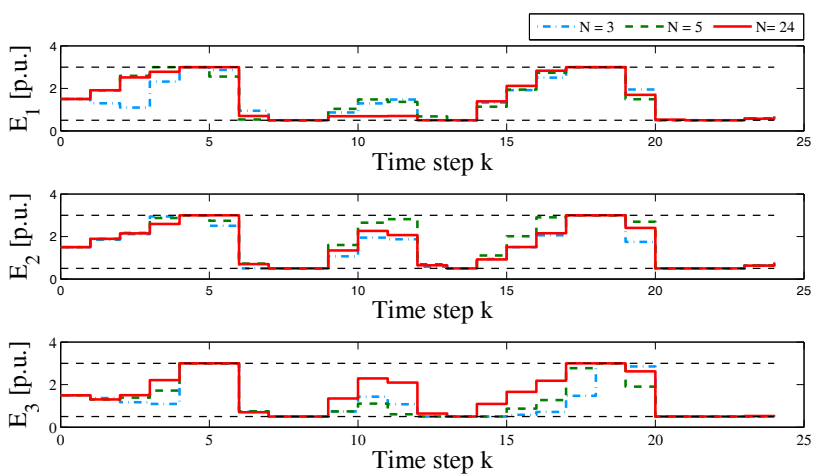

Fig. 6. Storage evolution over simulation horizon. Comparison of different lengths of prediction horizon $N=3, N=5, N=24$.

TABLE II

COMPARISON OF OPERATION COSTS, $N=5$

\begin{tabular}{|c|c|l|l|}
\hline$\mu \mathrm{CHP}$ & storage & costs [m.u.] & increase \\
\hline \hline$\sqrt{ }$ & $\sqrt{ }$ & $2.73 \cdot 10^{4}$ & base \\
\hline$\sqrt{ }$ & $\mathrm{x}$ & $2.98 \cdot 10^{4}$ & $9.2 \%$ \\
\hline $\mathrm{x}$ & $\sqrt{ }$ & $2.94 \cdot 10^{4}$ & $7.7 \%$ \\
\hline $\mathrm{x}$ & $\mathrm{x}$ & $3.07 \cdot 10^{4}$ & $12.5 \%$ \\
\hline
\end{tabular}

the heat load peaks and charged when no heat is required. However, the heat storage devices are not only important for the heat supply but indirectly also for electricity generation, since the $\mu \mathrm{CHP}$ devices can be operated according to the electricity load requirements due to the heat storage devices. At high electricity prices, electricity generation via $\mu \mathrm{CHP}$ is cheaper than via the generators, thus, the $\mu \mathrm{CHP}$ devices are preferably used for supplying the electricity demand while storing all excessive produced heat. This is also the reason why the contents of storages $\mathrm{E}_{1}$ and $\mathrm{E}_{2}$ rise again at the end of the simulation. During the heat load peaks almost all gas is directed into the furnaces because the thermal efficiencies of the $\mu \mathrm{CHPs}$ are not sufficient in order to supply the heat loads, not even by means of the heat storages. During these time periods, the operational costs increase correspondingly because the $\mu \mathrm{CHPs}$ can not be used for supplying the electricity loads.

In the following the operation costs are compared for different system setups regarding the $\mu \mathrm{CHPs}$ and the storage devices. As base case serves the configuration with $\mu \mathrm{CHPs}$ and storage devices. In Table II the increase in costs for the different cases are presented, in each case with a prediction horizon length of $N=5$. In the first two cases, the $\mu$ CHPs are utilized and the performance with and without heat storages is compared. Using the $\mu \mathrm{CHP}$ devices without the heat storages, total operation costs of $2.98 \cdot 10^{4}$ m.u. are obtained, corresponding to an increase of $9.2 \%$. This is due to the fact that the $\mu$ CHP devices cannot be utilized during periods without heat loads because the thereby produced heat cannot be used. The second two cases present the costs obtained in decoupled operation mode, namely when the electricity and natural gas networks are optimized independently of each other. No power is converted by the $\mu \mathrm{CHP}$ devices in this mode. Running the optimization without $\mu \mathrm{CHP}$ usage but including the heat 


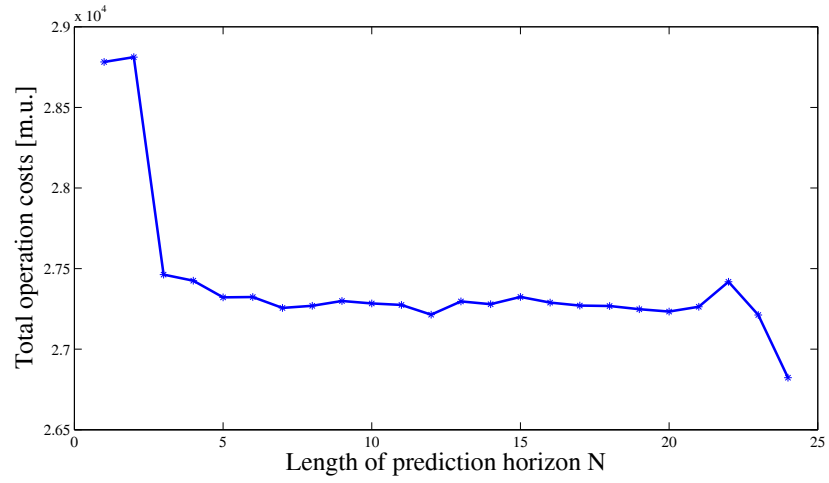

Fig. 7. Total operation costs for different lengths of prediction horizon $N$.

storages, total costs of $2.94 \cdot 10^{4} \mathrm{~m} . \mathrm{u}$. are obtained. Thus, by decoupling both infrastructures instead of operating them at once, generation costs are increased by $7.7 \%$. Running the simulation with either the $\mu \mathrm{CHP}$ nor the storage devices yields total costs of $3.07 \cdot 10^{4}$ m.u., corresponding to an increase of $12.5 \%$.

\section{Comparison between different lengths of prediction horizon}

For showing the effect of prediction, prediction horizons with different lengths $N$ are compared. In order to obtain a fair comparison, the prediction horizon is reduced towards the end of the simulation. Hence, in each case, the controller knows the same data, i.e., the measurements of the same 24 time steps. Figure 7 shows the total operation costs defined in (18) for different lengths of the prediction horizon $N$. Generally, the operation costs decrease with increasing prediction horizon. But this is not always the case. Depending on the input profiles, some prediction horizon lengths yield poorer results since the planned actions are suboptimal with respect to the whole simulation horizon. As can be seen, a fast decay of the operation costs occurs mainly within the first five prediction horizon lengths $N=1, \ldots, N=5$. For longer prediction horizons, not much reduction of the cost is gained, except for optimizing for all 24 time steps at once $(N=24)$. Besides that, computational effort increases with increasing prediction horizon length.

In Fig. 6, the storage contents for different lengths of prediction horizons $(N=3, N=5, N=24)$ are compared. At a prediction horizon with a length of $N=3$ (dash-dotted line), the storage devices are filled up too late or are even emptied before the heat load peaks (storage devices $\mathrm{E}_{1}$ and $\mathrm{E}_{3}$ at time steps $k=1 \ldots 3$ ) because the controller recognizes these peaks too late. With increasing length of prediction horizon, the storage devices are filled up earlier. By operating the storage devices in a more efficient way, i.e. mainly by filling them up earlier, the above discussed cost reductions with increasing prediction horizon length (Fig. 7) are achieved. Especially the storage device at hub $\mathrm{H}_{3}$ is utilized more efficiently with increasing prediction horizon length. Since hub $\mathrm{H}_{3}$ has no direct gas access, it avoids filling up the storage device when knowing only limited information in advance.

When optimizing for all 24 time steps at once (solid line) the most efficient behavior over the simulation horizon is obtained. The control variables for all next 24 time steps are determined and applied at time step $k$. But optimizing for all time steps at once is not applicable in practice since the data for the whole next day is normally not known in advance. Moreover, possibly occurring disturbances cannot be handled and computational effort becomes too high. Hence in practice, applying MPC with a properly chosen length of prediction horizon is the best choice. In general, depending on the specifications, a trade-off between control performance and computational effort has to be made. For the application example presented in this paper, a prediction horizon length of $N=5$ yields the best compromise. Moreover, issues such as obtainable forecasts and size of possible disturbances also influence the choice of an adequate length of prediction horizon.

\section{CONCLUSIONS AND FUTURE RESEARCH}

In this paper we have proposed the application of model predictive control to interconnected hub systems. The dynamics of storage devices, forecasts on energy prices and demand profiles, and operational constraints are taken into account adequately by the predictive control scheme, which is an effective control approach for this type of systems. The performance of different prediction horizons of varying length have been compared. With an increasing length of the prediction horizon total operation costs decrease, but computational effort increases accordingly. Future research will address the incorporation of disturbances within the profiles instead of assuming perfect forecasts. Furthermore, a distributed implementation of the central controller will be developed. In addition, network operators, which influence the energy exchanges between the hubs, are to be incorporated into the interconnected hub system.

\section{ACKNOWLEDGMENTS}

This research is supported by the project "Vision of Future Energy Networks" (VoFEN) of ABB, Areva T\&D, Siemens, and the Swiss Federal Office of Energy, the BSIK project "Next Generation Infrastructures (NGI)", the Delft Research Center Next Generation Infrastructures, the European STREP project "Hierarchical and distributed model predictive control (HDMPC)", and the project "Multi-Agent Control of Large-Scale Hybrid Systems" (DWV.6188) of the Dutch Technology Foundation STW.

\section{REFERENCES}

[1] J. Hernandez-Santoyo and A. Sanchez-Cifuentes, "Trigeneration: An alternative for energy savings," Applied Energy, vol. 76, no. 1-3, pp. 219-277, 2003.

[2] T. W. M. Shahidehpour, Y. Fu, "Impact of natural gas infrastrucutre on electric power systems," Proceedings of the IEEE, vol. 93, no. 5, pp. 1024 - 1056, 2005.

[3] M. Arnold, R. R. Negenborn, G. Andersson, and B. De Schutter, "Distributed control applied to combined electricity and natural gas infrastructures," in Proceedings of the International Conference on Infrastructure Systems, Rotterdam, The Netherlands, Nov. 2008.

[4] M. Geidl and G. Andersson, "Optimal power flow of multiple energy carriers," IEEE Transactions on Power Systems, vol. 22, no. 1, pp. 145155, 2007.

[5] J. M. Maciejowski, Predictive Control with Constraints. Harlow, England: Prentice Hall, 2002.

[6] E. F. Camacho and C. Bordons, Model Predictive Control. New York, New York: Springer-Verlag, 2004.

[7] P. Kundur, Power System Stability and Control. New York, New York: McGraw-Hill, 1994. 
[8] E. S. Menon, Gas Pipeline Hydraulics, New York: Taylor \& Francis, 2005.

[9] D. P. Bertsekas, Nonlinear Programming. Beltmore, Massachusetts: Athena Scientific, 2003.

[10] P. E. Gill, W. Murray, and M. A. Saunders, "SNOPT: An SQP algorithm for large-scale constrained optimization," SIAM Journal on Optimisation, vol. 12, no. 4, pp. 979-1006, 2002.

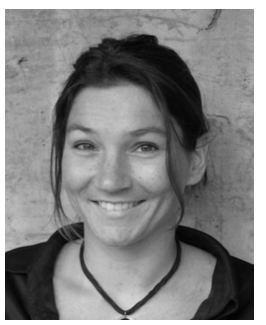

Michèle Arnold (S'07) was born in Bern, Switzerland. She received her MSc degree in electrical engineering and information technology from ETH Zurich (Swiss Federal Institute of Technology Zurich), Switzerland, in 2006.

In 2006, she joined the Power Systems Laboratory of ETH Zurich where she is working towards her $\mathrm{PhD}$. Her research interests are modeling and optimization of power systems, multi-agent systems and distributed control.

She is a student member of IEEE.

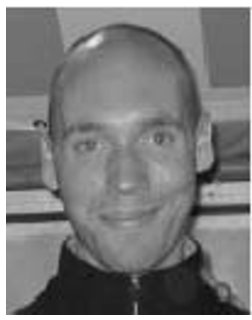

Rudy Negenborn was born in Utrecht, The Netherlands. He received the MSc degree in computer science (with a specialization in intelligent systems) cum laude from Utrecht University, The Netherlands, in 2003. In 2007, he obtained his $\mathrm{PhD}$ degree at the Delft Center for Systems and Control of the Delft University of Technology, The Netherlands. His $\mathrm{PhD}$ thesis was on multi-agent or distributed model predictive control with applications to power networks. Currently, Rudy Negenborn is a postdoctoral researcher at the same center.

Rudy Negenborn's more fundamental research interests include multi-agent systems, distributed control, model predictive control, and hybrid systems. His more applied research interests include applications to transportation networks in general, and water and power networks in particular.

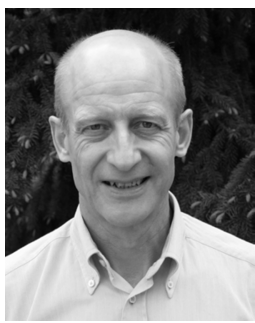

Göran Andersson (M'86, SM'91, F'97) was born in Malmö, Sweden. He obtained his MSc and PhD degree from the University of Lund in 1975 and 1980 , respectively.

In 1980 he joined ASEA, now ABB, HVDC division in Ludvika, Sweden, and in 1986 he was appointed full professor in electric power systems at the Royal Institute of Technology (KTH), Stockholm, Sweden. Since 2000 he is a full professor in electric power systems at ETH Zurich (Swiss Federal Institute of Technology Zurich), Switzerland. His research interests are in power system analysis, simulation and control. Another research interest is future energy and power systems.

$\mathrm{He}$ is a member of the Royal Swedish Academy of Engineering Sciences and Royal Swedish Academy of Sciences, and he is active in IEEE PES. He was the recipient of the IEEE PES Outstanding Power Educator Award 2007.

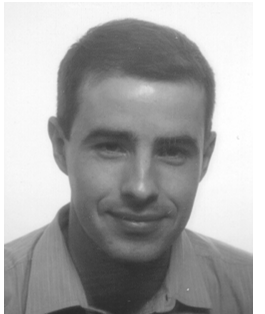

Bart De Schutter (M'08) was born in Ekeren, Belgium. He received the MSc degree in electrotechnical-mechanical engineering in 1991 and the $\mathrm{PhD}$ degree in Applied Sciences (summa cum laude with congratulations of the examination jury) in 1996, both at K.U.Leuven, Belgium. Currently, he is a full professor at the Delft Center for Systems and Control and at the Marine \& Transport Technology department of Delft University of Technology in Delft, The Netherlands.

Bart De Schutter was awarded the 1998 SIAM Richard C. DiPrima Prize and the 1999 K.U.Leuven Robert Stock Prize for his $\mathrm{PhD}$ thesis. He is associate editor of Automatica and of the IEEE Transactions on Intelligent Transportation Systems. His current research interests include hybrid systems control, discrete-event systems, multi-agent systems, control of intelligent transportation systems, and optimization. 